\title{
Module correspondence in finite groups
}

\author{
By Tetsuro Oкuyama \\ (Received February 25, 1980)
}

\section{Introduction}

The problem to investigate relations between the local and global properties of a finite group is one of the most important and elementary problems in the theory of finite groups. Concerning a prime number $p$, several techniques to investigate the global properties from $p$-local structure of groups are studied by many authors and play an important role for the advancement of the theory of finite groups (see [15]).

The representation theory of finite groups provides an effective tool to connect local properties to global properties. Hence for studies of the representation theory itself, it is also an important to investigate relations between the local and global properties. The result of Brauer and Fowler [5] is a representative example in this direction. They showed that there exist at most a finite number of simple groups with a specified centralizer of an involution. Several induction theorem by Artin, Brauer and others are also representative ones in this problem.

When we are interested in a prime $p$, the modular representation theory of finite groups with respect to $p$ is a refinement of the ordinary representation theory and at the same time is an effective tool to investigate $p$-local properties of finite groups. In studying it the theory of vertices and sources of Green, together with a series of studies of Brauer, has many contents to be developed in the future.

In this paper we shall investigate the theory of vertices and sources, especially the Green correspondence and try to refine several results in it for a certain type of finite groups.

In section 2, we are concerned with irreducible modules with trivial sources and investigate properties of the Green correspondence for them.

In section 3 , as an application of section 2 we define a family of finite groups called $M_{p}$-groups and study the representation theory of them.

In section 4 , we are mainly interested in finite solvable groups and some correspondence between a set of irreducible modules of a solvable group and its subgroup. This correspondence relates to the Green correspondence a little. 
In section 5, we try to give some refinement of Brauer's induction theorem in terms of $p$-blocks. Dress's induction theorem is also considered there.

Let $G$ be a finite group and $p$ a fixed rational prime number. In this paper we use the following notations and terminologies and for other notations and terminologies we shall refer to books of Gorenstein [16], Dornhoff [8] and Feit [11].

$R$; a complete discrete valuation ring with maximal ideal $(\pi) \ni p$.

$K$; the quotient field of $R$.

$F ;=R /(\pi)$, the residue field of $R$ which has characteristic $p$.

$R[G], K[G], F[G]$; the group algebras of $G$ over $R, K$ and $F$ respectively.

We assume that fields $K$ and $F$ are both splitting fields for all groups considered in this paper. All modules considered are right unital and finitely generated.

$V^{N}$; the induced module for an $F[H]$-module $V$ to $N$ where $H$ and $N$ are subgroups of $G$ with $H \subseteq N$.

$V_{\mid H}$; the restriction of an $F[N]$-module $V$ to $H$ where $H$ and $N$ are subgroups of $G$ with $H \subseteq N$.

$V^{x}$; the conjugate module of an $F[H]$-module $V$ by an element $x$ in $G$ which is an $F\left[H^{x}\right]$-module.

If $H$ is a normal subgroup of $G$, we define the inertia subgroup of $V$, denoted by $I_{G}(V)$, to be the set $\left\{x \in G \mid V^{x} \cong V\right\}$.

$V \mid W$; for $F[G]$-modules $V$ and $W, V$ is isomorphic to a direct summand of $W$.

In the above we use the same notations for $R[T]$-modules too for a subgroup $T$ of $G$.

$L_{0}(G)$; an $F[G]$-module of dimension 1 on which $G$ acts trivially.

For $F[G]$-modules $V$ and $W$ and a subgroup $H$ of $G$ define $(V, W)_{H}=$ $\operatorname{Hom}_{F[H]}(V, W)$. The relative norm map $T_{H, G} ;(V, W)_{H} \rightarrow(V, W)_{G}$ is defined as follows. $T_{H, G}(\lambda)=\sum_{x} \lambda^{x}$ for $\lambda \in(V, W)_{H}$, where $x$ ranges over a set of representatives of right cosets of $H$ in $G$ and $\lambda^{x}$ is the map $v \rightarrow\left(\lambda\left(v x^{-1}\right)\right) x$. If $\mathfrak{S}_{\mathfrak{Z}}$ is a set of subgroups of $G$, we define $(V, W)_{G}^{\mathfrak{S}}=(V, W)_{G} /(V, W)_{\mathscr{S}, G}$ where $(V, W)_{\mathfrak{5}, G}=\sum_{H \in \mathfrak{5}} \operatorname{Im} T_{H, G}$.

Now we shall describe the Green correspondence which is frequently used in the paper. We refer to a book of Feit [11].

Let $V$ be an indecomposable $F[G]$-module. Then there exists a $p$ subgroup $D$ which is a minimal subgroup of $G$ such that $V$ is $F[D]$-projective. A group $D$ is uniquely determined up to conjugation by an element 
in $G$ and called a vertex of $V$. If $D$ is a vertex of $V$, then here exists an indecomposable $F[D]$-module $W$ such that $V \mid W^{G}$. $W$ is also determined up to conjugation by an element in $N_{G}(D)$.

Let $D$ be a $p$-subgroup of $G$ and $N$ a subgroup of $G$ with $N_{G}(D) \cong N$. Define the following sets of subgroups;

$\mathfrak{X}(G, D, N)=\mathfrak{X}=\left\{A \mid A \subseteq D \cap D^{x}\right.$ for some $\left.x \in G-N\right\}$

$\mathfrak{Y}(G, D, N)=\mathfrak{Y}=\left\{A \mid A \subseteq N \cap D^{x}\right.$ for some $\left.x \in G-N\right\}$

$\mathfrak{A}(G, D, N)=\mathfrak{A}=\{A \mid A \subseteq D$ and $A \notin \mathfrak{X}\}$.

The Green correspondence $f$ with respect to $(G, D, N)$ is the map from the set of indecomposable $F[G]$-modules with vertices in $\mathfrak{A}$ to the set of those of $F[N]$-modules. For properties of the Green correspondence, see [11].

\section{Irreducible modules with trivial sources}

In this section we shall consider properties of the Green correspondence of irreducible modules with trivial sorces. First we shall prove the following which is concerned with the Brauer characters afforded by modules with trivial sources. For a definition of the Brauer characters see [8].

Lemma 2.1. Let $G$ be a finite group, $V$ an indecomposable $F[G]$ module with vertex $D$ and $\beta$ the Brauer character afforded by $V$. Assume the p-part of $\operatorname{dim}_{F} V$ is equal to one of $|G: D|$. Then we have that $\mid G: C_{G}(x)$ $\mid \beta(x) / \beta(1)$ lies in $R$ for every $p^{\prime}$-element $x$ in $G$.

Proof. Let $Q$ be a Sylow $p$-subgroup of $C_{G}(x)$. The result of Green (Theorem 10 in [17]) shows that $\beta(x) /\left|Q: Q \cap D^{y}\right|$ lies in $R$ for each element $y$ in $G$. By our assumption the $p$-part of $\left|G: C_{G}(x)\right| / \beta(1)$ is equal to $|D| /|Q|$ and therefore the lemma follows as $|D| /|Q| \cdot|Q| /\left|Q \cap D^{y}\right|$ is a positive power of $p$.

Lemma 2.2. Let $G$ be a finite group and $V$ an irreducible $F[G]$ module with vertex $D$. Let $f$ be the Green correspondence with respect to $\left(G, D, N_{G}(D)\right)$. If a source of $V$ is trivial one, then $f(V)$ is irreducible and the p-part of $\operatorname{dim}_{F} V$ is equal to one of $|G: D|$.

Proof. Let $W=f(V)$ and $N=N_{G}(D)$. In the notation defined in section $1(V, V)_{G} \cong F$ as $V$ is irreducible and therefore $(V, V)_{G}^{\mathfrak{E}} \cong F$. Thus $(W, W)_{N}^{\vec{b}} \cong F$ by the result of Green ([19]). Let $E \in \mathfrak{Y}$, then $E D \neq E$. Let $T$ be a set of representatives of right cosets of $E$ in $E D$. Since $W$ has a vertex $D, D \triangleleft N$ and has a trivial source, $D$ acts on $W$ trivially and $W$ is a projective indecomposable $F[N / D]$-module. Let $\lambda$ be any $F[E]$-endmorphism of $W$ and put $\mu=T_{E, E D}(\lambda)$. Then for each element $w$ in $W$ we have $\mu(w)=\sum_{t \in T}\left(\lambda\left(w t^{-1}\right)\right) t=$ 
$\sum_{t \in T} \lambda(w)=0$. Thus by the definition of $(W, W)_{N}^{\vec{B}}$, we have that $(W, W)_{N} \cong F$. Then since $W$ is projective as an $F[N / D]$-module, we have that $W$ is irreducible. Since $W$ is projective and irreducible as an $F[N / D]$ - module, there exists an $R$-free $R[N / D]$-module $U$ such that $W=U / U(\pi)$ and $U \otimes K$ is irreducible. Thus the $p$-part of $\operatorname{dim}_{F} W$ is equal to one of $|N: D|$. By a property of Green correspondence we have $W^{G}=V \oplus X$, where $X$ is an $F[G]$-module whose indecomposable direct summands all have vertices properly contained in $D$. By Corollary 2 in [17] $\operatorname{dim}_{F} X$ is divisible by $p \cdot|G: D|$. Thus the last statement of the lemma follows and the proof of the lemma is complete.

TheOREm 2.3. Let $G$ be a finite group and $L$ an irreducible $F[G]$ module with vertex $D$. If a source of $L$ is trivial, then we have the following ;

(1) Let $f$ be the Green correspondence with respect to $\left(G, D, N_{G}(D)\right)$. Then $f(L)$ is irreducible and has $D$ as a vertex. In particular, $N_{G}(D) / D$ and $D C_{G}(D) / D$ have a p-block of defect 0 .

(2) If $\varphi$ is the Brauer character afforded by $L$, then $\left|G: C_{G}(x)\right| \varphi(x) / \varphi(1)$ lies in $R$ for every $p^{\prime}$-element $x$ in $G$.

(3) If $B$ is a p-block of $G$ which contains $L$, then we can take a $p$ subgroup $D_{0}$ as a defect group of $B$ so that $Z\left(D_{0}\right) \subseteq Z(D) \subseteq D \cong D_{0}$.

(4) If $N_{G}(D) / D$ is abelian, then $D=D_{0}$ in the statement (3).

Proof. Let $N=N_{G}(D)$ and $C=D C_{G}(D)$. The first half of the statement (1) follows from Lemma 2.2. As $f(L)$ is irreducible and projective as an $F[N / D]$-module, a $p$-block of $N / D$ which contains $f(L)$ is of defect 0 and so the second half of (1) is proved. The statement (2) follows from Lemma 2.1 and 2.2. Let $b$ be a $p$-block of $N$ which contains $f(L)$ and $b_{1}$ a $p$ block of $C$ covered by $b$. Let $W$ be an indecomposable direct summand of $f(L)_{1 G}$ contained in $b_{1}$. Then $W$ is irreducible and projective as an $F[C / D]$-module. Thus $b_{1}$ has $D$ as a defect group by Lemma 64.3 in [8] and $b_{1}^{N}=b$ by Lemma 64.8 in [8]. By the result of Nagao (see Lemma 56.5 in [8]) we have $b^{G}=B$. Then the statement (3) follows from the result of Brauer [3]. Finally we shall prove the statement (4). Our proof is based on the idea of Erdman in [10]. Set $T=I_{N}(W)$ and $T_{1}$ be a subgroup of $T$ such that $T_{1} / C$ is a Sylow $p$-subgroup of $T / C$. Then $W$ can be extended to an $T\left[T_{1}\right]$-module $W_{1}$ uniquely by [11] and therefore $W_{1} \mid f(L)_{\mid T_{1}}$ as $T_{1}$ is normal in $N$. Since $f(L)$ has $D$ as a vertex we have that $T_{1} / C=1$ and therefore $\left|I_{N}(W): C\right|$ is a $p^{\prime}$-number. Hence by the extension of Brauer's first main theorem (see $\S 64$ in [8]) $b$ has a defect group $D$ and then the statement (4) follows from Brauer's first main theorem. 


\section{Monomial irreducible modules}

Definition 3.1. Let $X$ be an $F[G]$-module. Then $X$ is called monomial if $X=Y^{G}$, where $Y$ is an $F[H]$-module with $\operatorname{dim}_{F} Y=1$ for some subgroup $H$ of $G$.

Since for a $p$-group $P$ only one dimensional $F[P]$-module is trivial one, we have the following elementary fact for a monomial module;

REMARK 3.2. If $X$ is a monomial $F[G]-m o d u l e$, then every indecomposable summand of $X$ has a trivial source.

Proposition 3.3. Let $X$ be an irreducible $F[G]$-module with vertex $D, N=N_{G}(D)$ and $f$ the Green correspondence with respect to $(G, D, N)$. If $X$ is monomial, then we have the following;

(1) $f(X)$ is irreducible and monomial,

(2) $\operatorname{dim}_{F} f(X)$ divides $\operatorname{dim}_{F} X$.

Proof. Since $X$ has a trivial source by the above remark we can apply Theorem 2.3 for $X$ and then we have that $f(X)$ is irreducible. Let $H$ be a subgroup of $G$ and $Y$ an $F[H]$-module with $\operatorname{dim}_{F} Y=1$ such that $Y^{G}=X$. We may assume that $D \cong H$. By the Mackey decomposition it follows that $Y^{G}{ }_{\mid N}=\otimes \sum_{x}\left(Y^{x}{ }_{\mid H^{x} \cap N}\right)^{N}$, where $x$ ranges over a complete set of representatives of $(H, N)$-double cosets in $G$. Taking $x=1 \quad Y_{\left.\right|_{N_{H^{(D)}}}}$ is a direct summand of $X_{\mid N}$. From the fact that $D \subseteq \operatorname{Ker} Y$ and $D \triangleleft N$ every indecomposable summand of $Y_{\mid N_{l}(D)^{N}}$ has $D$ as a vertex. Then by a property of the Green correspondence $Y_{\mid N,(D)}{ }^{N}=f(X)$ and therefore $f(\mathrm{X})$ is monomial. Then $\operatorname{dim}_{F} f(X)=\left|N: N_{H}(D)\right|$ and it devides $|G: H|=\operatorname{dim}_{F} X$. Thus the proposition is proved.

Definition 3.4. A finite group $G$ is called an $M_{p}$-group if every irreducible $F[G]$-module is monomial.

A finite group $G$ is called an $M$-group if every irreducible complex character of $G$ is induced from a linear character of some subgroup of $G$. Properties of $M$-groups are studied by several authors and it is well known that $M$-groups are solvable (see $\S 15$ in [8]).

In the remaining in this section we shall investigate properties of $M_{p^{-}}$ groups.

REMARK 3.5. (1) An $M$-group is an $M_{p}$-group for every prime number $p$. This follows the fact that $M$-groups are solvable and the result of Fong-Srwan (§ 72 in [8]).

(2) $S L(2,3)$ is an $M_{2}$-group since it is 2-closed and has an abelian 2-complement. But it is known that $S L(2,3)$ is not an M-group (see 
Exercise 15.1 in [8]). So $M_{p}$-groups need not be M-groups.

(3) Subgroups of $M_{p}$-groups need not be $M_{p}$-groups. A finite group defined in Exercise 15.2 in [8] provides a such example.

(4) If $P$ is normal p-subgroup of $G$ and $G / P$ is an $M_{p}$-group, then $G$ is also an $M_{p}$-group.

Definition 3.6. Let $G$ be a finite group and put $G^{p(0)}=G$. For each positive integer $i$ define $\left.G^{p(i)}=O^{p}\left[G^{p(i-1)}, G^{p(i-1)}\right]\right)$.

$G^{p(i)}$ is a characteristic subgroup of $G$ and $O^{p}\left(G^{p(i)}\right)=G^{p(i)}$ for each integer $i>0$.

The following theorem will be proved by the similar method as those of the proof of Theorem 5.12 in [26].

THEOREM 3.7. Let $G$ be an $M_{p}$-group and let $1=d_{1}<\cdots<d_{k}$ be the distinct dimensions of the irreducible $F[G]$-modules. Let $X$ be an irreducible $F[G]$-module with $\operatorname{dim}_{F} X=d_{i}$. Then we have that $G^{p(i)} \leqq \operatorname{Ker} X$.

Proof. If $i=1$, then $\operatorname{dim}_{F} X=1$ and the theorem is proved easily in this case. Assume that $i>1$ and we shall prove the theorem by induction on $i$. If $V$ is an irreducible $F[G]$-module with $\operatorname{dim}_{F} V<\operatorname{dim}_{F} X$, then $\operatorname{dim}_{F} V=d_{j}$ for some positive integer $j<i$ and $G^{p(i-1)} \subseteq G^{p(j)} \cong \operatorname{Ker} V$ by induction. Choose a subgroup $H$ of $G$ and an $F[H]$-module $Y$ with $\operatorname{dim}_{F}$ $Y=1$ such that $Y^{G}=X$. By our assumption $|G: H|>1$. If $V$ is any irreducible $F[G]$-module which appears in a composition factor of $L_{0}(H)^{G}$, then $\operatorname{dim}_{F} V<|G: H|=\operatorname{dim}_{F} X$ since $L_{0}(G)$ is contained in $L_{0}(H)^{G}$. Thus since $G^{p(i-1)}$ is generated by $p$-regular elements and contained in the kernel of each irreducible constituent of $L_{0}(H)^{G}, G^{p(i-1)} \cong \operatorname{Ker} L_{0}(H)^{G} \subseteq H$. Then we have that $G^{p(j)} \subseteq O^{p}\left(H^{\prime}\right)$. Since $O^{p}\left(H^{\prime}\right) \leqq \operatorname{Ker} Y$ and $G^{p(i)} \triangleleft G$ it follows that $G^{p(i)} \subseteq \operatorname{Ker} X$. Thus the proof of the theorem is complete.

Corollary 3.8. If $G$ is an $M_{p}$-group, then $G$ is solvable.

It is not true that all solvable groups are $M_{p}$-groups as a group $\operatorname{SL}(2,3)$ shows. SL $(2,3)$ is solvanle but is not an $M_{3}$-group for it has an irreducible module of dimension two over an algebraically closed field of characteristic 3 and has no subgroup of index 2. However, Dade has shown that every finite solvable group is a subgroup of an $M$-group (see Satz. V. 18.11 in [21]) and therefore every finite solvable group is contained in an $M_{p}$-group.

THEOREM 3.9. Let $G$ be an $M_{p}$-group and $B$ a p-block of $G$ with defect group $D$. Let $N=N_{G}(D)$ and $b$ a p-block of $N$ reith $b^{G}=B$ and $f$ the Green correspondence with respect to $(G, D, N)$. Then $f$ defines a oneto-one correspondence between the set of all irreducible $F[G]$-modules in $B$ with vertex $D$ and the set of all irreducible $F[N]$-modules in $b$. If 
furthermore $D$ is abelian, the number of irreducivle $F[G]$-modules in $B$ and the number of those of $b$ are equal.

Proof. If $D$ is abelian, every irreducible $F[G]$-module in $B$ has a vertex $D$ by Theorem 2.3. (3). So the second assertion of the theorem follows from the first. Let $X_{1}$ and $X_{2}$ be irreducible $F[G]$-modules in $B$ with vertex $D$. Then a property of the Green correspondence shows that $f\left(X_{1}\right) \cong f\left(X_{2}\right)$ if and only if $X_{1} \cong X_{2}$. If we can show that the number of irreducible $F[G]$-modules in $B$ with vertex $D$ and the number of irreducible $F[N]$-modules in $b$ are equal, then from Proposit!on 3.3. (1) it follows that $f$ defines a desired one-to-one correspondence. So it will suffice to show that

(*) Let $G$ be a finite solvable group and $B, D, N$ and $b$ as in Theorem 3. 9. Then the number of irreducible $F[G]$-modules in $B$ with vertex $D$ is equal to the number of irreducible $F[N]$-modules in $B$.

We shall show this assertion in the next section (see Theorem 4. 1).

Corollary 3.10. Let $G$ be an $M_{p}$-group and $D$ a p-subgroup of $G$. If $b$ is a p-block of $N=N_{G}(D)$ with defect group $D$, then every irreducible $F[N]$-module in b is monomial. In particular, if $D$ is a Sylow p-subgroup of $G$, then $N$ is an $M_{p}$-group.

Proof. The first assertion of the corollary follows from Proposition 3.3. (1) and Theorem 3. 9. The second follows from the first and the fact that every $p$-block of $N$ has $D$ as defect group if $D$ is a Sylow $p$ subgroup of $G$.

Corollary 3.11. If $G$ is an $M$-group with a Sylow p-subgroup $P$. If $H$ is a p-complement of $N_{G}(P)$, then $H$ is an M-group.

Proof. By Remark 3.5. (1) $G$ is an $M_{p}$-group and then by Corollary 3. $10 H \cong N_{G}(P) / P$ is also an $M_{p}$-group. Since $p \nmid|H|$ it follows that $H$ is an $M$-group and the proof of the corollary is complete.

\section{Module correspondence in finite groups}

In this section we shall investigate properties of the Green correspondence in finite solvable groups and construct some correspondence between a set of irreducible modules of a solvable group and a set of those of its subgroup. Very important studies of the modular representation theory of finite solvable groups are done by Fong in his papers [12] and [13]. We shall use his results frequently in this section. The results of Hamernik and Michler [21] and Cliff [6] also provide useful tools for our investigation. 
As a final consequences of our consideration in this section we shall prove the following;

THEOREM 4.1. Let $G$ be a finite solvable group and $B$ a p-block of $G$ with defect group $D$. Let $N=N_{G}(D)$ and $b$ a p-block of $N$ with $b^{G}=B$. Then the number of irreducible $F[G]$-modules in $B$ with vertex $D$ is equal to the number of irreducible $F[N]$-modules in $b$.

Let $G$ be a finite group and $H$ a normal subgroup of $G$. If $W$ is an irreducible $F[H]$-module, then the investigation of $F[G]$-modules whose restriction to $H$ contain $W$ is very important in studying the representation theory. Also if $V$ is an $F[G]$-module it is so to study the restriction of $V$ to $H$. First in this section we shall study these problems in connection with our applications to the case considered in later in this section. In the case characteristic of $F$ is 0 , our results that we shall described are studied by several authors and well known (see [25], [26] and [28]). Our proof of them will be done with references to [25] and [26].

Lemma 4.2. Let $G$ be a finite group, $H$ a subgroup of $G$ and $N$ a normal $p^{\prime}$-subgroup of $G$ such that $G=H N$. Put $M=H \cap N$. Let $X$ be an irreducible $F[N]$-module such that $X_{\perp M}$ is also irreducible and $I_{G}(X)$ $=G$. Then the map $\sigma ; V \mapsto V_{\mid H}$ is a one-to-one correspondence between the set of all irreducible $F[G]$-modules $V$ with $X \mid V_{\mid N}$ and the set of all ir-

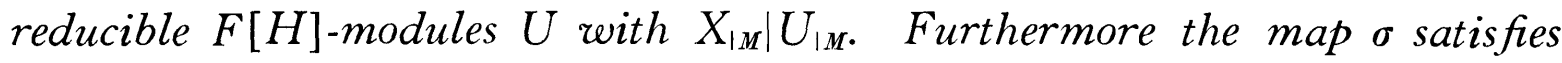
the following;

(1) If $V$ is an irreducible $F[G]$-module with $X \mid V_{\mid N}$, then $V$ and $\sigma(V)$ have a common vertex.

(2) If $B$ is a p-block of $G$ which covers p-block $\{X\}$ of $N$, then $\sigma(B)$ is also a p-block of $H$ which covers a p-block $\left\{X_{\mid M}\right\}$ of $M$ and $B$ and $\sigma(B)$ have a common defect group.

(3) In (2) if furthermore a defect group $D$ of $B$ satisfies that $N_{G}(D)$ $\subseteq H$, then $B=\sigma(B)^{G}$.

Proof. If $T$ is a subgroup and $W_{1}$ and $W_{2}$ are $F[T]$-modules, then we denote $I_{T}\left(W_{1}, W_{2}\right)=\operatorname{dim}_{F} \operatorname{Hom}_{F[T]}\left(W_{1}, W_{2}\right)$. Let $Y=X_{\mid M}$. Let $V$ be as in the statement (1) and $U$ an irreducible submodule of $V_{\mid H}$. Then we have that $V_{\mid N}=n X$ and $U_{\mid M}=m Y$ for some positive integer $n$ and $m$. We shall show that $n=m$ which implies that $V_{I H}=U$ is irreducible. It follows easily that $n \geqq m$ by counting dimensions of $V$ and $U$. Since $X$ and $Y$ are irreducible, $n=I_{N}\left(V_{\mid N}, X\right)$ and $m=I_{M}\left(U_{\mid M}, Y\right)$. Applying the Frobenius reciplocity low and Mackey decomposition theorem we have $m=I_{N}\left(\left(U_{1 M}\right)^{N}, X\right)=$ $I_{N}\left(U^{G}{ }_{I N}, X\right)=I_{G}\left(U^{G}, X^{G}\right) . \quad V$ is isomorphic to a factor module of $U^{G}$ and $X^{G}$ 
is projective as $N$ is of $p^{\prime}$-order. So we have that $I_{G}\left(U^{G}, X^{G}\right)=I_{G}\left(V, X^{G}\right)=$ $I_{N}\left(V_{\mid N}, X\right)=n$. Thus we have $n=m$. Since $H$ is of $p^{\prime}$-index in $G$, it is clear that $V$ and $U$ have a common vertex. As $Y^{N}=X \oplus W$, where $W$ is $F[N]$-module which has no irreducible submodule isomorphic to $X, V$ is a unique indecomposable component of $\left(V_{\mid H}\right)^{G}$ which has an irreducible $F[N]$-submodule isomorphic to $X$. Thus for irreducible $F[G]$-modules $V_{1}$ and $V_{2}$ with $X \mid V_{i \mid N}, V_{1} \cong V_{2}$ if and only if $V_{1 \mid H} \cong V_{2 \mid H}$. As $I_{G}(X)=G$ we have that for an irreducible $F[H]$-module $U$ with $Y \mid U_{\mid M}, U^{G}=V \oplus W$ where $V$ is an $F[G]$-module such that $V_{\mid N}=n X$ and $W$ is an $F[G]$-module not containing an $F[N]$-submodule isomorphic to $X$. Then it is shown by the similar argument in the above that $V$ is irreducible. Thus $\sigma$ is a one-to-one correspondence. Let $W_{i}, 1 \leqq i \leqq s$, be all non-isomorphic projective indecomposable $F[G]$-modules with $X \mid W_{i \mid N}$. Let $Z_{j}, 1 \leqq j \leqq s$, be all non-isomorphic projective indecomposable $F[H]$-modules with $Y \mid Z_{j \mid M}$. Let $V_{i}$ be an irreducible $F[G]$-module which corresponds to $W_{i}$, that is, a unique irreducible factor module of $W_{i}$. Let $U_{j}$ be one of $Z_{j}$ as $V_{i}$ for $W_{i}$. Let $X^{G}=$ $\oplus \sum_{i} a_{i} W_{i}$ and $Y^{H}=\oplus \sum_{j} b_{j} Z_{j}$. Then $a_{i}=I_{G}\left(X^{G}, V_{i}\right)$ and $b_{j}=I_{H}\left(Y^{H}, Z_{j}\right)$. Then the above argument implies that after suitable renumbering $a_{i}=b_{i}$ and $W_{i \mid H}=Z_{i}$. Thus every projective indecomposable $F[G]$-module $W$ is also indecomposable as $F[H]$-modules.

Let $B$ be a $p$-block as in the statement (2) and $V_{1}$ and $V_{2}$ be irreducible $F[G]$-modules in $B$. We shall show that $\sigma\left(V_{1}\right)$ and $\sigma\left(V_{2}\right)$ are in the same $p$-block of $H$. By Theorem 46.2 in [8] there exist projective indecomposable $F[G]$-modules $W_{1}, \cdots, W_{n}$ such that $W_{i}$ and $W_{i+1}$ have a common irreducible constituent and $V_{1}$ appears in a composition factor of $W_{1}$ and $V_{2}$ in one of $W_{n}$. So we may assume that $V_{1}$ and $V_{2}$ appear in the same projective indecomposable $F[G]$-module as its irreducible constituents. The the result follows from the preceding paragraph as every projective indecomposable $F[G]$-module is also indecomposable as $F[H]$-module. Thus the statement (2) is proved.

Let $B$ be a $p$-block of $G$ with defect group $D$ such that $N_{G}(D) \cong H$. Then there is a unique $p$-block $B_{1}$ of $H$ by Brauer's first main theorem such that $B_{1}$ has a defect group $D$ and $B_{1}{ }^{G}=B$. Let $V$ be an irreducible $F[G]$ module in $B$ with vertex $D$, then $V_{1 H}{ }^{G}=V \oplus W$ for some $F[G]$-module $W$ and $V_{\mid H}$ and $V$ are in correspondence by the Grren correspondence with respect to $(G, D, H)$. Then the result of Green (Theorem 5.8 in [20]) shows that $V_{1 H}$ is in $B_{1}$ and therefore the statement (3) follows.

In the following we shall state the results of Fong and Cliff which is needed in later. 
Theorem 4.3 (Fong [12], Cliff [6]). Let $G$ be a finite group, $N$ a normal p-subgroup of $G$ and $X$ an irreducible $F[N]$-module. Let $T=I_{G}(X)$. If. $B$ is a p-block of $G$ which covers a p-block $\{X\}$ of $N$, then there exists a p-block $B^{\prime}$ of $T$ such that

(1) There is a one-to-one correspondence between irreducible $F[G]$ modules $L$ in $B$ and irreducible $F[T]$-modules $L^{\prime}$ in $B^{\prime}$ given by $L=L^{\prime a}$. If $L$ and $L^{\prime}$ are in this correspondence, then they have a common vertex.

(2) $B$ and $B^{\prime}$ have a common defect group.

Corollary 4.4. Let $G, H, N$ and $M$ be as in Lemma 4.2. Assume furthermore that $M$ is normal in $G$. Let $Y$ be an irreducible $F[M]$ module with $I_{G}(Y)=H$. Then $Y^{N}$ is irreducible and the map $\tau ; U \mapsto U^{G}$ is a one-to-one correspondence between the set of irreducible $F[H]$-modules $U$ with $Y \mid U_{\mid M}$ and the set of irreducible $F[G]$-modules $V$ with $Y^{G} \mid V_{\left.\right|_{N}}$. Furthermore the map $\tau$ satisfies the following;

(1) If $U$ is an irreducible $F[H]$-module with $Y \mid U_{\mid M}$, then $U$ and $\tau(U)$ have a common vertex.

(2) If $b$ is a p-block of $H$ which covers a p-block $\{Y\}$ of $M$, then $\tau(b)$ is a p-block of $G$ which covers a p-block $\left\{Y^{G}\right\}$ of $N . \quad b$ and $\tau(b)$ have a common defect group. In fact $b^{G}$ is defined and $b^{G}=\tau(b)$.

Proof. Since $I_{N}(Y)=M$, it follows that $Y^{N}$ is irreducible by Satz 2.3 in [24]: Then the corollary is proved using Theorem 4.3.

THEOREM 4.5 (Fong [12], Cliff, [6]). In Theorem 4.3 assume that $T=G$. Then there is a finite group $\hat{G}$ with a central cyclic $p^{\prime}$-subgroup $A$ such that $\hat{G} / A=G / N$ and there is a p-block $\hat{B}$ of $\hat{G}$ which satisfies the following;

(1) There is a one-to-one correspondence between irreducible $F[\hat{G}]$ modules $\hat{L}$ in $\hat{B}$ and irreducible $F[G]$-modules $L$ in $B$ such that $\hat{L}$ and $L$ have isomorphic vertices.

(2) $\hat{B}$ and $B$ have isomorphic defect groups.

Lemma 4.6. Let $G, N, X$ and $T$ be as in Theorem 4.3 and assume $T=G$. Let $B$ be a p-block of $G$ which covers a p-block $X$ of $N$. Let $D$ be a defect group of $B, H$ a subgroup of $G$ containing $N$ and $N_{G}(D)$ and $b$ a p-block of $H$ with defect group $D$ such that $b^{G}=B$. Then there is a subgroup $\hat{H}$ of $\hat{G}$ such that $\hat{H} / A=H / N$ and there is a p-block $\hat{b}$ of $\hat{H}$ with the following properties;

(1) $\hat{b}$ satisfies the conclusions of Theorem 4.5 for $H, N$, and $b$.

(2) $\hat{b}^{\hat{\theta}}$ is defined and $\hat{b}^{\hat{\theta}}=\hat{B}$ where $\hat{B}$ is a p-block of $\hat{G}$ in Theorem 4.5 for $B$. 
Proof. The lemma can be proved by using the argument of Cliff in section 2 in [6]. Let $\hat{H}$ be a subgroup of $\hat{G}$ such that $\hat{H} / A \cong H / N$ under the isomorphism $\hat{G} / A \cong G / N$ in Theorem 4.5. Since $H$ and $b$ satisfies the conditions in Theorem 4.5, the existence $\hat{b}$ such that $\hat{b}$ satisfies (1) is clear. If $V$ and $W$ are $F[G]$-modules which contain an $F[N]$-module isomorphic to $X$ and $W \mid V$, then the construction of $F[\hat{G}]$-mudules $\hat{W}$ and $\hat{V}$ defined by Cliff in [6] from $W$ and $V$ shows that $\hat{W} \mid \hat{V}$. Also it holds that if $U$ is an $F[H]$-module which contains $X$ as an $F[N]$-submodule, then $\widehat{U^{a}}=$ $\hat{U}^{\hat{t}}$. Take an indecomposable $F[H]$-module $U$ with vertex $D$ and let $W$ be an $F[G]$-module which corresponds to $U$ in the Green correspondence with respect to $(G, D, H)$. Then $\hat{W}$ corresponds to $\hat{U}$ in the Green correspondence with respect to $(\hat{G}, \widehat{D N}, \hat{H})$. Then the result of Green in [20] shows that $\hat{b}^{\hat{\theta}}=\hat{B}$ and the lemma is proved.

The following two propositions are proved using a proof of Wolf of Theorem 2.2 and 2.3 in [28].

Proposition 4.7. Let $G$ be a finite group and $H$ and $E$ be a subgroup of $G$. Assume the following;

(1) $G$ is a semidirect product $E H$ and $E \triangleleft G$,

(2) $1 \varsubsetneqq Z(E) \subseteq Z(G), Z(E)$ is cyclic and $E$ is a $p^{\prime}$-group.

(3) $E / Z(E)$ is an elementary abelian q-group for some prime $q$ distinct from $p$.

(4) $[E / Z(E), L]=E / Z(E)$ for some $C_{H}(E) \triangleleft L \triangleleft H$ such that $L / C_{H}(E)$ is a p-group.

(5) $X$ is a faithful irreducible $F[E]$-module.

Let $Y$ be an irreducible $F[Z(E)]$-submodule of $X_{\mid Z(E)}$. Then we have the following;

(i) There exists an irreducible $F[G]$-module $Z$ with the following properties; (a) $Z_{\mid E}=X$, (b). $C_{H}(E) \subseteq \operatorname{Ker} Z$ and (c). if $Z^{*}=\operatorname{Hom}_{F}(Z, F)$, the dual module of $Z$, then $Z \otimes Z^{*}=L_{0}(G) \oplus W$, where a vertex of every indecomposable direct summand of $W$ does not contain a Sylow p-subgroup of $L E$.

(ii) If $U$ is an $F[H]$-module, then there is a unique $F[G]$-module $U^{0}$ with $\operatorname{Ker} U^{0} \supseteqq E$ and $U^{0}{ }_{1 H}=U$. Then the map $\mu ; U_{\mapsto} \rightarrow U^{0} \otimes Z$ is a oneto-one correspondence between the set of irreducible $F[H]$-modules and the set of irreducible $F[G]$-modules which contain $X$ as an $F[E]$-submodule. The map $\mu$ satisfies the following; (a). if $U$ is an irreducible $F[H]$-module, then $U$ and $\mu(U)$ have a common vertex, (b). if $b$ is a p-block of $H$, then $\mu(b)$ is a p-block of $G$ which covers a p-block $\{X\}$ of $E$, (c). if $U$ is an irreducible $F[H]$-module, then $U \otimes U^{*} \mid \mu(U) \otimes \mu(U)^{*}$. 
Proof. First we shall prove the statement (i). By the result of Wolf (Theorem 2.2 in [28]) there exists an irreducible $F[G]$-module $Z$ which satisfies (a) and (b) in (i). Put $V=Z \otimes Z^{*}$. Then $V$ is an $F\left[G / Z(E) C_{H}(E)\right]$ module. As $E$ is of $p^{\prime}$-order we have that $V=C_{V}(E) \oplus[V, E]$, where $C_{V}(E)$ is a submodule of $V$ centralized by $E$ and $[V, E]$ is a submodule of $V$ generated by all elements $v x-v$ for $v$ in $V$ and $x$ in $E$. As $E$ is normal in $G, C_{V}(E)$ and $[V, E]$ are both $F[G]$-modules. By our assumption (4) $L$ acts on $E / Z(E)$ fixed-point-freely and therefore a vertex of every indecomposable $F\left[G / Z(E) C_{H}(E)\right]$-module not containing $E$ in its kernel does not contain a Sylow $p$-subgroup of $L E$. Since $Z$ is irreducible $C_{V}(E)=C_{V}(G)$ and it has an $F$-dimension 1 . So $Z$ has a property (c) in (i). Thus the statement (i) is proved. By Satz 2.4 and 2.5 in [24] if $U$ is an irreducible $F[H]$-module then $U^{0} \otimes Z$ is irreducible and if $W$ is a projective indecomposable $F[H]$-module then $W^{0} \otimes Z$ is also projective and indecomposable. Then the statement (ii) except for (c) is proved in the similar manner with that of Lemma 4.2. The statement (c) in (ii) follows from (c) in (i).

Proposition 4.8. Let $G$ be a finite group and $E, H$ subgroups of $G$. Assume the following;

(1) $G=E H, E \triangleleft G, E \cap H=Z(E) \subseteq Z(G)$ and $E$ is a $p^{\prime}$-group,

(2) $Z(E) \neq 1$ is cyclic,

(3) $E / Z(E)$ is an elementary abelian q-group for some prime number $q$ distinct from $p$,

(4) $[L, E / Z(E)]=E / Z(E)$ for some $C_{H}(E) \leqq L \triangleleft H$ such that $L / C_{H}(E)$ is a p-group.

(5) $Y$ is a faithful irreducible $F[Z(E)]$-module.

Let $X$ be an irreducible summand of $Y^{E}$, then the following hold;

(i) There is a one-to-one correspondence $\alpha$ between the set of all $p$ blocks of $G$ which cover a p-block $\{X\}$ of $E$ and whose defect groups contain a Sylow p-subgroup of $L$ and the set of p-blocks of $H$ which covers a p-block $\{Y\}$ of $Z(E)$ and whose defect groups contain a Sylow p-subgroup of $L$. Furthermore $\alpha$ is the map which preserves defect groups.

(ii) There is a one-to-one correspondence $\beta$ between the set of irreducible $F[G]$-modules $V$ with $X \mid V_{\mid E}$ and the set of irreducible $F[H]$-modules $U$ with $Y \mid U_{\mid Z(E)}$. If $V$ has a vertex containing a Sylow p-subgroup of $L$, so has $\beta(V)$ and in that case $V$ and $\beta(V)$ have a common vertex.

(iii) If $V$ is in a p-block $B$ of $G$, then $\beta(V)$ is in a p-block $\alpha(B)$ of $H$.

Proof. This proposition is proved by using the method of Wolf in [28] and Proposition 4.7. Thus we shall omit the details of the proof. 
Now we shall prove the following which is used to prove the theorem described in the begining in this section.

Proposition 4.9. Let $G$ be a finite group with a Sylow p-subgroup $P$. Let $E$ be a normal $p^{\prime}$-subgroup of $G$ such that $E N_{G}(P)=G$. Set $N=$ $N_{G}(P)$ and $M=N \cap E$. If $X$ is an irreducible $F[E]$-module with $I_{G}(X)=G$, then the following hold.

(1) There exists a unique $F[M]$-module $Y$ such that $I_{M}\left(X_{\mid M}, Y\right) \not \equiv 0$ $(\bmod p)$. This $Y$ is $N$-invariant.

(2) Assume G/PE is abelian. Then $X$ is extendible to $G$ if and only if $Y$ is extendible to $N$.

(3) The number of all irreducible $F[G]$-modules whose restrictions to $E$ contain $X$ is equal to the number of all irreducible $F[N]$-modules whose restrictions to $M$ contain $Y$.

Proof. (1) is the result of Glauberman (see $\S 13$ in [25]). In fact Glauberman's correspondence is a 1-lcorrespondence between P-invariant irreducible $F[E]$-modules $V$ and irreducible $F[M]$-modules $U$ such that $I_{M}\left(V_{\mid M^{\prime}} U\right) \not \equiv 0(\bmod p)$.

Next we shall prove the statement (2). Notice that if $X$ is extendible to a $p$-complement of $G$, then so is to $G$. This follows from the result of Fong (Lemma 2. A, [13]) since $G / E$ is $p$-closed.

(2) is proved by induction on $|G|$. Let $L$ be a $p$-complement in $N$. As $L / M$ is abelian, there is a subgroup $A$ with $M \subseteq A \subseteq L$ such that $L / A$ is cyclic and $C_{P / P^{\prime}}(A) \neq 1$. Assume $C_{P / P^{\prime}}(A)=P / P^{\prime}$. Then $C_{P}(A)=P$. If $Y$ is extendible to $N$, then so is to $L$. Let $\hat{Y}$ be an extension of $Y$ to $L$ and $Y_{0}$ be the restrivtion of $\hat{Y}$ to $A$. The number of irreducible $F[A]$ modules whose restrictions to $M$ contain $Y$ is equal to $|A / M|$ and such irreducibles are all $P$-invariant since $A \subseteq C_{G}(P)$. Then by the Glauberman's correspondence it follows that the number of irreducible $F[A E]$-modules whose restrictions to $E$ contain $X$ is also equal to $|A / M|=|A E / E|$. Since $A E / E$ is abelian, this implies that such irreducibles are all extensions of $X$ to $A E$. Let $X_{0}$ be the irreducible $F[A E]$-module which corresponds to $Y_{0}$ in the Glauberman correspondence. This $X_{0}$ is an extension of $X$ to $A E$ and $I_{L E}\left(X_{0}\right)=L E$ since $I_{L}\left(Y_{0}\right)=L$. Thus $X_{0}$ is extendible to $L E$ as $L E / A E$ $\cong L / A$ is cyclic (Satz 3.1, [27]). And therefore $X$ is extendible to $G$ since $L E$ is a $p$-complement of $G$. Conversely if $X$ is extendible to $G$, then by the similar argument as in the above it follows that $Y$ is extendible to $N$.

Next assume $C_{P / P^{\prime}}(A)=Q / P \neq P / P^{\prime}$. As $A$ is normal in $L, Q$ and $Q E$ are normal in $N$ and $G$ respectively. Let $H=Q L E$ and $J=C_{E}(Q)$. Then there is a unique irreducible $F[J]$-module $W$ such that $I_{J}\left(W, X_{I_{J}}\right) \equiv 0(\bmod p)$ 
by Glauberman's correspondence. This $W$ is $N_{G}(Q)$-invariant and $I_{M}\left(Y, W_{\mid M}\right)$ $\not \equiv 0(\bmod p)$. Considering the group $N_{G}(Q) / Q$ we have by induction that $Y$ is extendible to $N$ if and only if $W$ is extendible to $N_{G}(Q)$. Also by induction we have that $W$ is extendible to $N_{H}(Q)$ if and only if $X$ is extendible to $H$. As $|G: H|$ and $\left|N_{G}(Q): N_{H}(Q)\right|$ are powers of $p$ we can conclude that $Y$ is extendible to $N$ if and only if $X$ is extendible to $G$. Thus the statement (2) is proved.

Since $|P E: E|$ is a power of $p, X$ is extended to $P E$ uniquely. As $E$ and $G / P E$ are of $p^{\prime}$-order we can apply the result of Gallagher (see 11, [25] Problem 11.7 and 8) and (3) follows from (2). Thus the proposition is proved.

Now we can prove the theorem which is described in the begining in this section.

THEOREM 4.1. Let $G$ be a finite p-solvable group and $B$ a p-block of $G$ with defect group $D$. Let $N=N_{G}(D)$ and $b$ a p-block of $N$ such that $b^{a}=B$. Then the number of irreducible $F[G]$-modules in $B$ with vertex $D$ is equal to the number of irreducible $F[N]$-modules in $b$.

Proof. We shall prove the theorem by induction on the order of $G$. If $O_{P}(G)=P \neq 1$, then $D \supseteqq P$ and every irreducible $F[G]$-module has $P$ in its kernel. Put $\bar{G}=G / P$. It is clear that $N_{\bar{G}}(\bar{D})=\overline{N_{G}(D)}$. Let $B_{1}, \cdots, B_{k}$ be all $p$-blocks of $\bar{G}$ such that $B_{i} \subseteq B$ and has a defect group $D$. Let $b_{1}, \cdots, b_{s}$ be all $p$-blocks of $\bar{N}$ with $b_{j} \subseteq b$. Then by Brauer's first main theorem $k=s$ and after suitable renumbering $b_{i}^{\vec{G}}=B_{i}$. By induction the theorem holds for $b_{i}$ and $B_{i}$. If an irreducible $F[G]$-module with vertex $D$ is in a $p$-block of $G$ with defect group $D$, then as considered as an $F[\bar{G}]$-module it is in a $p$-block of $\bar{G}$ with defect group $\bar{D}$. Thus the theorem follows in this case. So we may assume that $O_{p^{\prime}}(G)=E \neq 1$. Assume that $E D \not G$. Then $H=N_{G}(D) E$ is a proper subgroup of $G$. Let $B_{0}$ be a unique $p$-block of $H$ with $B_{0}{ }^{G}=B$ and $b^{H}=B_{0}$. As the theorem holds for $B_{0}$ and $b$ by induction, it will suffice to show that the number of irreducible $F[G]$ modules in $B$ with vertex $D$ and the number of those in $B_{0}$ are equal. Let $X$ be an irreducible $F[E]$-module such that a $p$-block $\{X\}$ is covered by $B$. If $I_{G}(X) \neq G$, then by induction and Theorem 4.3 the result follows. If $I_{G}(X)=G$, then by Theorem 4.5 and Lemma 4.6 we may assume that $O_{p^{\prime}}(G)$ is central and therefore $O_{p}(G) \neq 1$. Thus in this case the result also follows. Next assume that $E D \triangleleft G$. Let $X$ be as in the above. If $I_{G}(X) \neq G$, then by induction and Theorem 4.3 our theorem holds. If $I_{G}(X)=G$, then $D$ is a Sylow $p$-subgroup of $G$ by arguments of Fong [12] and $B$ is a unique $p$-block of $G$ which covers a $p$-block $\{X\}$. Then the theorem follows from Proposition 4.9 and the proof of the theorem is complete. 


\section{Induction theorems of Brauer and Dress}

Let $G$ be a finite group. For a subgroup $H$ of $G$, let $C(H)$ denote the character ring of $H$. It is the set of all integral linear combinations of characters which are afforded by $K[H]$-modules. If $H$ and $N$ are subgroups of $G$ such that $H \subseteq N$, we define $C(H)^{N} \subseteq C(N)$ to be the set of elements in $C(N)$ which are induced from those in $C(H)$.

In [4] Brauer has shown the following;

Theorem (Brauer [4]). Let $G$ be a finite group and $\mathcal{E}$ the set of all elementary subgroups of $G$. Then we have

$$
C(G)=\sum_{E \in \mathcal{E}^{\mathcal{B}}} C(E)^{G} .
$$

First in this section we shall investigate this theorem of Brauer in block-wise and prove the following;

TheORem 5.1. Let $G$ be a finite group and $B$ a p-block of $G$ with defect group $D$. Let $C_{B}(G)$ be the set of all integral linear combinations of characters in $B$. Let $\mathcal{E}(D)$ be the set of all elementary subgroups of $G$ whose Sylow p-subgroups are contained in some conjugates of $D$. Then

$$
C_{B}(G) \underset{E_{\in \in \mathcal{E}^{\prime}(D)}}{\subseteq} C(E)^{G} .
$$

This theorem will be obtained as a corollary of Theorem 5.3 in later.

Lemma 5.2. Let $H=P \times A$ be a finite group where $P$ is a p-group and $A$ is a $p^{\prime}$-group. Let $V$ be an indecomposable $R$-free $R[H]$-module with vertex $D \subseteq P$ and $\chi$ a character afforded by $V$. Then there exists $\eta$ a character afforded by some $K[D \times A]$-module such that $\chi=\eta^{H}$.

Proof. By III. $\S 3$ in [11] we may assume that $V$ is absolutely indecomposable and there exist indecomposable R-free $R[H]$-modules $U$ and $W$ such that $P$ is in the kernel of $W, A$ is in the kernel of $U$ and $V=$ $U \otimes W$. It is obvious that $U$ has $D$ as a vertex and therefore it will be suffice to show the lemma in the case $H=P$. Then by a result of Green (see III. $\S 3$ in [11]) we may assume that there exists an indecomposable $R$-free $R[D]$-module $V_{1}$ such that $V_{1}{ }^{P}=V$. Thus the proof of the lemma is complete.

If $B$ is a $p$-block of $G$ with defect group $D$, then every $R[G]$-module which lies in $B$ is $R[D]$-projective and has a vertex contained in $D$ (see Theorem 54.1 in [8]). So Theorem 5.1 is obtained as a corollary of the following. 
THEOREM 5.3. Let $V$ be an $R$-free $R[G]$-module which is $R[D]$ projective with $D$ a p-subgroup and $\chi$ a character afforeded by $V$. Then we have

$$
\chi \in \sum_{E \in \mathcal{E}(D)} C(E)^{G} .
$$

Proof. By Brauer's induction theorem we have $1_{G}=\sum_{E \in \epsilon^{\dot{e}}} a_{E} \theta(E)^{G}$ where $a_{E}$ is an integer and $\theta(E)$ is a character of $E . \quad \theta(E)$ is afforded by some $R$-free $R[E]$-module $U(E)$ by [11]. Since every indecomposable direct summand of $U(E) \otimes V_{\mid E}$ has a vertex contained in some conjugate of $D$ by [11] and $E$ is a finite group of type as in Lemma 5.2, the theorem follows.

It is known that there exists an irreducible complex character of $G$ contained in $B$ such that a $p$-part of its degree is that of $|G: D|$. Thus we have the following;

REMARK 5.4. Let $G, B$ and $D$ as in Theorem 5. 1. If $C_{B}(G) \underset{E \in \mathcal{E}^{\prime}\left(D_{0}\right)}{\in} C(E)^{G}$ for some $D_{0} \subseteq D$, then $D_{0}=D$.

In the remainder of this section we assume that the field $F$ is algebraically closed. For a subgroup $H$ of $G$ let $A(H)$ denote the representation algebra of $F[H]$ over the ring of rational integers. It is defined as follows. $A(H)$ is the abelian group generated by the set of all isomorphism classes $(V)$ of $F[H]$-modules $V$ subject to the relations $\left(V_{1} \oplus V_{2}\right)=\left(V_{1}\right)+\left(V_{2}\right)$. If $H$ and $N$ are subgroups of $G$ such that $H \subseteq N$, we define $A(H)^{N} \cong A(N)$ to be the set of elements in $A(N)$ which are induced from those in $A(H)$.

Dress has shown in [9] the following which asserts that for the representation algebra of a finite group an induction theorem of type of Brauer's induction theorem also holds.

TheOREm (Dress [9]). Let $G$ be a finite group and $\varepsilon_{p}$ be the set of all subgroups $E$ of $G$ such that $E / O_{p}(E)$ is an elementary group of $p^{\prime}$ order. Then we have

$$
A(G)=\sum_{E \in \mathcal{E}_{p}} A(E)^{G} .
$$

As we can prove Theorem 5.1, we shall be able to prove the following theorem in connection with Theorem of Dress.

THEOREM 5.5. Let $G$ be a finite group and $B$ a p-block of $G$ with defect group $D$. Let $A_{B}(G)$ denote the set of all integral linear combinations of $F[G]$-modules which lie in $B . \quad$ Let $\mathcal{E}_{p}(D)$ be the set of all subgroups $E$ of $G$ which lie in $\mathcal{E}_{p}$ such that $O_{p}(E)$ is contained in some conjugate of $D$. Then we have 


$$
A_{B}(G) \subseteq \sum_{E \in \mathcal{E}_{p}^{(D)}} A(E)^{G} .
$$

Theorem 5.5 follows from Theorem 5.8 which is proved in later.

Lemma 5.6. Let $H$ be a solvable p-closed finite group with a $p$ complement $A$ and a Sylow p-subgroup $P$. Let $V$ be an indecomposable $F[H]$-module with vertex $D$ such that $D$ is normal in $H$. Then there exists an $F[A D]$-module $W$ such that $V=W^{H}$.

Proof. We shall prove the lemma by induction on the order of $H$. If $H=P$, then the lemma follows from the result of Green [17]. Let $U$ be an indecomposable $F[D]$-module such that $V \mid U^{H}$. Put $T=I_{H}(U)$, the inertia subgroup of $U$ in $H$. By Satz 2.2 in 24 there exists an indecomposable $F[T]$-module $V_{0}$ such that $V_{0}^{H}=V$. If $T \neq H$, then by induction we have our lemma and therefore we may assume that $T=H$. Then the lemma follows from the following Lemma 5.7.

Lemma 5.7. Let $H, A, P, V$ and $D$ be as in Lemma 5.6. Let $U$ be an indecomposable $F[D]$-module which is a source of $V$. If $I_{H}(U)=H$, then there exists an $F[A D]$-module $W$ such that $W^{H}=V$. Furthermore $W$ is a unique indecomposable $F[A D]$-module which satisfies that $V \mid W^{H}$.

Proof. If $H=P$, then by the result of Green [17] we have that $V=U^{P}$. If $U^{\prime}$ is another indecomposable $F[D]$-module such that $V \mid U^{\prime P}$, then $V=U^{\prime P}$ and therefore $U^{\prime}=U$ as $I_{P}(U)=P$. So we shall prove the lemma by induction on the order of $H$. Since $A$ is solvable we can take a normal subgroup $A_{0}$ of $A$ with prime index, say $q$. As $A$ is of $p^{\prime}$-order, $q$ is distinct from $p$. Put $T=A D, T_{0}=A_{0} D$ and $H_{0}=T_{0} P$. Let $V_{0}$ be an indecomposable $F\left[H_{0}\right]$-module such that $V_{0} \mid V_{\mid H_{0}}$. Then we have the following two cases;

(1) $V=V_{0}^{H}$ and $I_{H}\left(V_{0}\right)=H_{0}$,

(2) $V_{I_{0}}=V_{0}$ and $I_{H}\left(V_{0}\right)=H$.

Proof in the case (1). We have that $V_{\mid H_{0}}=\oplus \sum_{x} V_{0}^{x}$, where $x$ ranges over a set of representatives of right cosets of $H_{0}$ in $H$. We can take all $x$ 's in $T$. By induction there exists an $F\left[T_{0}\right]$-module $W_{0}$ such that $W_{0}^{H_{0}}=V_{0}$. By a uniqueness of the existence of $W_{0}$ we have the $I_{T}\left(W_{0}\right)=T_{0}$ and $W_{0}^{T}$ is indecomposable by Satz 2.2 in [24]. Then $W=W_{0}^{T}$ satisfies that $W^{H}=V$. Let $W^{\prime}$ be another indecomposable $F[T]$-module which satisfies that $V \mid W^{\prime H}$. Then by the fact that $V_{0}\left|V_{\mid H_{0}}\right| W^{\prime H_{\mid H_{0}}}=\left(W_{\mid T_{0}}\right)^{H_{0}}, W_{0}$ is a direct summand of $W^{\prime}{ }_{1 T_{0}}$ and therefore $W^{\prime}=W_{0}^{T}$ as $W^{\prime}$ is indecomposable and $I_{T}\left(W_{0}\right)=T_{0}$. Thus the lemma is proved in this case. 
Proof in the case (2). Since $H / H_{0}$ is cyclic and $I_{H}\left(V_{0}\right)=H$. it follows that $V_{0}{ }^{H}=V_{1} \oplus V_{2} \oplus \cdots \oplus V_{q}$ and $V_{i}$ 's are all non-isomorphic and indecomposable. We set $V=V_{1}$. By induction there exists and $F\left[T_{0}\right]$-module $W_{0}$ such that $W_{0}{ }^{H_{0}}=V_{0}$. We have that $I_{T}\left(W_{0}\right)=T$ since $I_{H}\left(V_{0}\right)=H$ and the existence of $W_{0}$ is unique. Then by Satz 3.1 in [24] there exist $F[T]$ modules $W_{1}, W_{2}, \cdots, W_{q}$ such that $W_{i \mid T_{0}}=W_{0}, W_{0}^{T}=W_{1} \oplus W_{2} \oplus \cdots \oplus W_{q}$ and $W_{i}$ 's are all non-isomorphic and indecomposable. Thus we have that $W_{1}{ }^{H} \oplus W_{2}{ }^{H} \oplus \cdots \oplus W_{q}^{H}=V_{1} \oplus V_{2} \oplus \cdots \oplus V_{q}$ and after suitable renumbering $W_{i}{ }^{H}=V_{i}$ for each $i$. For $i=1, W=W_{1}$ satisfies that $W^{H}=V$. Let $W^{\prime}$ be another indecomposable $F[T]$-module such that $V \mid W^{\prime H}$. Then the fact that $V_{0}\left|V_{\mid H_{0}}\right|\left(W^{\prime H}\right)_{\mid H_{0}}=\left(W_{\mid T_{0}}\right)^{H_{0}}$ implies that $W_{0} \mid W^{\prime}{ }_{\mid K_{0}}$. So as $W^{\prime}$ is indecomposable $W^{\prime}=W_{i}$ for some $i, 1 \leqq i \leqq q$, and therefore $W^{\prime}=W_{1}=W$. Thus the proof of the lemma is complete.

Now we can prove the following from which Theorem 5.5 follows.

Theorem 5.8. Let $G$ be a finite group and $V$ an indecomposable $F[G]$-module with vertex $D$. Then we have

$$
V \in \sum_{E \in \mathcal{E}_{p}(D)} A(E)^{G} .
$$

Proof. If $D=1$, then the theorem follows from the result of Dress [9]. So we shall proved the theorem by induction on the order of $D$. By a property of the Green correspondence $V=W^{a} \oplus U$, where $W$ is an indecomposable $F\left[N_{G}(D)\right]$-module with vertex $D$ and $U$ is an $F[G]$-module whose indecomposable direct summands all have vertices properly contained in $D$. Thus we may assume that $G=N_{G}(D)$, that is, $D$ is normal in $G$. By Dress's induction theorem $L_{0}(G)=\sum_{E \in \mathcal{E}_{p}} a_{E} V(E)^{G}$, where $a_{E}$ is an integer and $V(E)$ is an indecomposable $F[E]$-module. Since every indecomposable direct summand of $V(E) \otimes V_{\mid E}$ has a vertex contained in $D$ and $E$ is a $p$ closed solvable, our theorem follows from Lemma 5.6.

If $B$ is a $p$-block of $G$ with defect group $D$, then every indecomposable $F[G]$-module which lies in $B$ is $F[D]$-projective and has a vertex contained in $D$. Thus Theorem 5.5 follows from Theorem 5.8.

As is remarked in Remark 5.4, we have the following;

Remark 5.9. Let $G, B$, and $D$ be as in Theorem 5.5. If $A_{B}(G)$ $\subseteq \sum_{E \in \mathcal{E}_{p}\left(D_{0}\right)} A(E)^{G}$ for some $D_{0} \subseteq D$, then $D_{0}=D$. 


\section{References}

[1] J. L. Alperin: The main problem of block theory. in Proceedings of the Conference on Finite Groups edited by W. R. Scott and F. Gross. Academic Press, New York-San Francisco-London, 1976.

[2] R. BRAUER: Zur Darstellungstheorie der Gruppen von endlicher Ordnung. I, II. Math. Zeitschr. 63 (1956), 406-444, 72 (1959/60), 25-46.

[3] R. BRAUER: On blocks and sections in finite groups. I, II. Amer. J. Math. 89 (1967), 1115-1136, 90 (1968), 895-925.

[4] R. BRAUER: A characterization of the characters of groups of finite order. Ann. of Math. (2) 57 (1953), 357-377.

[5] R. Brauer and K. A. Fowler: On groups of even order. Ann. of Math. (2) 62 (1955), 565-583.

[6] G. H. ClifF : On modular representations of p-solvable groups. J. Algebra 47 (1977), 129-137.

[7] C. W. CURTIS and I. REINER: Representation Theory of Finite Groups and Associative Algebras. Wiley-Interscience, New York, 1962.

[8] L. DoRnhoff: Group Representation Theory. A, B. Marcel Dekker, New York, 1971.

[9] A. W. M. DREsS: Modules with trivial sources, modular monomial representations and a modular version of Brauer's induction theorem. Abh. Math. Sem. Univ. Hamburg, 44 (1975), 101-109.

[10] K. ERdmanN: Blocks and simple modules with cyclic vertices. Bull. London Math. Soc. 9 (1977), 216-218.

[11] W. FEIT: Representations of Finite Groups. Yale University, 1969 (mimeographed note).

[12] P. Fong: On the characters of $p$-solvable groups. Trans. Amer. Math. Soc. 98 (1961), 263-284.

[13] P. Fong: Solvable groups and modular representation theory. Trans. Amer. Math. Soc. 103 (1962), 484-494.

[14] G. Glauberman: Fixed points in groups with operator groups. Math. Zeitschr. 84 (1964), 120-125.

[15] G. Glauberman: Global and local properties of finite groups. in Finite Simple Groups edised by M. B. Powell and G. Higman. Academic Press, LondonNew York, 1971.

[16] D. GoREstein: Finite Groups. Harper and Row, New York, 1968.

[17] J. A. GREen: On the indecomposable representations of a finite group. Math. Zeitschr. 70 (1958/9), 430-445.

[18] J. A. GREEN : Blocks of modular representations. Math. Zeitschr. 79 (1962), $100-115$.

[19] J. A. GREEN: A transfer theorem for modular representations. J. Algebra 1 (1964), 73-84.

[20] J. A. GReEN: Relative module categories for finite groups. J. Pure Appl. Algebra 2 (1972), 371-393. 
[21] W. HAmernik and G. Michler: On vertices of simple modules in p-solvable groups. Mittelungen aus dem Math. Seminar Giessen, 121 (1976), 147-162.

[22] B. Huppert : Endliche Gruppen. I. Springer Verlag, Berlin-New York, 1967.

[23] B. Huppert : Bemerkungen zur modularen Darstellungstheorie, 1. Arch. Math. 26 (1975), 242-249.

[24] B. Huppert and W. Willems: Bemerkungen zur modularen Darstellungstheorie, 2. Arch. Math. 26 (1975), 486-496.

[25] I. M. IsAACs: Characters of solvable and symplectic groups. Amer. J. Math. 95 (1973), 594-635.

[26] I. M. IsAACs: Character Theory of Finite Groups. Academic Press, New York, 1976.

[27] W. Willems : Bemerkungen zur modularen Darstellungstheorie, 3. Arch. Math. 26 (1975), 497-503.

[28] T. R. Wolf: Characters of $p$-degree in solvable groups. Pacific I : [ath. 74 (1978), 267-271.

Department of Mathematics

Osaka City University 